ISSN 0103-5665 • 83

\title{
TOXICODEPENDÊNCIA E MATERNIDADE: UMA REVISÃO DE LITERATURA
}

\author{
DRUG ADDICTION AND MATERNITY: A LITERATURE REVIEW \\ DROGODEPENDENCIA Y MATERNIDAD: UNA REVISIÓN DE LITERATURA \\ Sofia A. Silva* \\ António P. Pires** \\ Maria J. Gouveia***
}

\section{RESUMO}

Pretende fazer-se uma revisão da literatura acerca da parentalidade de mães toxicodependentes, abordando as 1) consequências da toxicodependência na maternidade e 2) as condiçóes ambientais e contextuais dos pais toxicodependentes, e suas famílias de origem. A literatura atual aponta para um comportamento parental perturbado das mães toxicodependentes, embora saliente a importância da gravidez e maternidade como fator predisponente ao início de um tratamento e recuperação. São referidos efeitos disruptivos na saúde, nascimento e desenvolvimento pós-natal das crianças, na qualidade do vínculo e da interação mãe-filho, bem como repercussóes que se refletem na inadequação de cuidados maternais e risco aumentado de negligência e abuso. A investigaçáo focaliza a atenção em algumas áreas específicas do comportamento parental, verificando-se lacunas e resultados nem sempre consistentes, falta de estudos contemplando a relação direta entre abuso de substâncias e disfunçôes familiares e da parentalidade, bem como a experiência e dificuldades inerentes à parentalidade nesta situação de risco. É feita uma análise da investigação atual e fornecidas algumas diretrizes para futuras investigaçōes.

Palavras-chave: toxicodependência; gravidez; maternidade.

* ISPA - Instituto Universitário de Ciências Psicológicas, Sociais e da Vida, Lisboa, Portugal. ** ISPA - Instituto Universitário de Ciências Psicológicas, Sociais e da Vida, Lisboa, Portugal. *** ISPA - Instituto Universitário de Ciências Psicológicas, Sociais e da Vida, Lisboa, Portugal. 


\section{Abstract}

This article is a literature review on parenting of drug addicted mothers, addressing 1) the consequences of drug abuse in maternity and 2) the environmental conditions of drug addicted parents, and their families of origin. The current literature points to a disturbed parental behaviour of drug addicted mothers, although it stresses the importance of pregnancy and maternity as a predisposing factor to the beginning of a recovery treatment. Authors refer disruptive effects on health, birth and post-natal children development, on attachment quality and on mother-child interaction, as well as inadequate maternal care and high risk of abuse and negligence. The research focuses attention on specific areas of parental behavior, in which we can find gaps and incoeherences, characterized by the lack of studies covering the direct link between substance abuse and family dysfunction and parenting, as well as the experience and difficulties of parenting in the situation of risk that is drug addiction. We make an analysis of the current investigation and suggest some guidelines for future research.

Keywords: addiction; pregnancy; motherhood.

\section{RESUMEN}

El objetivo de la investigación es elaborar una revisión de la literatura sobre la parentalidad de las madres drogodependientes abordando las 1) consecuencias de la drogodependencia en la maternidad y 2) las condiciones ambientales y contextuales de los padres drogodependientes, y sus familias de origen. La literatura actual se orienta hacia un comportamiento parental perturbado de las madres drogodependientes, aunque realce la importancia del embarazo y maternidad como factor predisponente al inicio de un tratamiento y recuperación. Se refieren efectos disruptivos en la salud, nacimiento y desarrollo de los recién nacidos en el puerperio, en la calidad del vínculo e interacción madre-hijo, así como repercusiones que se reflejan en la inadecuación de los cuidados maternales y riesgo incrementado de negligencia y abuso. La investigación se focaliza en algunas áreas específicas del comportamiento parental, verificándose fallos y resultados que no son siempre consistentes, falta de estudios que reflejen la relación directa entre el abuso de substancias y disfunciones familiares y de la parentalidad, así como la experiencia y dificultades inherentes a la parentalidad en esta situación de riesgo. Se hace un análisis de la investigación actual y se facilitan algunas directrices para futuras investigaciones.

Palabras clave: drogodependencia; embarazo; maternidad. 


\section{Introdução}

A relação dos pais com os filhos, cuidados precoces e a sua continuidade e qualidade, têm sido evidenciados em vários estudos, pela sua importância na determinação e influência no desenvolvimento psicológico, social e afetivo da criança. Segundo Pires (2001) o equilíbrio emocional da criança depende em grande medida de um comportamento parental adequado, isto é, da sensibilidade dos pais na interaçáo com os filhos, da capacidade para perceberem os seus desejos e necessidades, manifestas ou não, e atenderem a essas necessidades adequadamente ao longo das diferentes fases do desenvolvimento. Por vezes, a transição para a parentalidade acontece num período ou situação de vida inesperada, em contextos menos favoráveis com alteraçóes significativas na vida dos pais (Limede, 2014). Quando o comportamento parental náo responde de forma adequada às solicitaçóes da criança, representando um risco para a sua saúde física e psicológica, torna-se uma prioridade a especial atenção às interaçóes da díade mãe-criança. Uma destas situações de risco, que reúne condições para se tornar um contexto problemático, é o caso das mães toxicodependentes, existindo diversos estudos que consideram o comportamento parental em toxicodependentes, perturbado, deficiente, frágil e com consequências nefastas para a criança e sua relaçáo com os pais (Mayes \& Truman, 2002), bem como um problema de saúde pública (Anthony, Austin, \& Cormier, 2010).

Este artigo pretende fazer uma revisão da literatura acerca da parentalidade de mães toxicodependentes, focando sobretudo em drogas como cannabis, heroína (ou metadona) e cocaína, dado serem escassas as revisóes de literatura sistematizadas e actualizadas acerca da parentalidade no consumo de drogas, sobretudo sobre os efeitos diretos da toxicodependência na criança e comportamento parental das mães, bem como sobre a importância da intergeracionalidade e condiçóes ambientais/familiares neste contexto. Verifica-se uma multiplicidade de fatores intervenientes na problemática da toxicodependência materna, pelo que se torna pertinente uma revisão dos estudos existentes em algumas áreas específicas que torne possível apontar linhas lacunares de investigação que permitam esclarecer aspectos concretos e guiar a intervenção para resultados mais eficazes. Assim, procuramos organizar esta revisão de literatura com enfoque em duas áreas principais, com vista à sistematização e compreensão geral das seguintes temáticas: 1) consequências da toxicodependência na maternidade e 2) condiçôes ambientais e famílias de origem dos pais toxicodependentes. 


\section{Método}

Foi realizada uma busca intensiva utilizando as bases de dados ERIC, PsycINFO, PsycARTICLES, Academic Search Complete, PEP Archive e Psychology and Behavioral Sciences Collection. As palavras chave utilizadas foram drug addicted mothers, substance abuse, parenting e child e os critérios de inclusão foram: analisado por especialistas e publicação nos últimos cinco anos.

\section{Resultados}

Um total de 269 publicaçóes foram revistas, tendo sido de entre estas e de outras de data anterior, selecionadas 59 e incluídas neste estudo, com base na sua relevância, temas menos estudados nos últimos cinco anos e atualidade dos estudos. Os temas desses artigos foram identificados e agrupados de acordo com sete categorias principais: comportamento parental em situação de risco, toxicodependência materna e patologia psicológica, consequências da toxicodependência na maternidade, condições ambientais das mães toxicodependentes, famílias de origem dos pais toxicodependentes, revisóes de literatura. Foi identificado um outro tema, mas não foi selecionado para inclusão: modelos e projetos de intervenção, uma vez que se verificou a existência de revisóes de literatura recentes sobre essa temática.

\section{Consequências da toxicodependência na maternidade}

A questão da maternidade na mulher toxicodependente é considerada particularmente preocupante dadas as potenciais implicaçóes nos filhos e até mesmo nas geraçóes que se seguem (Guerreiro, 2001). De facto, a capacidade de cuidar de um filho pode ser afectada por factores como a continuação do consumo na gravidez e maternidade, a que se associam as características de personalidade e a percepção que a mãe tem, realista ou distorcida, do comportamento da criança e/ ou por um estilo de vida marcado pelo stress, isolamento, dificuldades económicas e problemas legais (Xavier \& Paúl, 1997). Surge com frequência na literatura a associação entre a toxicodependência materna e a presença de perturbaçóes psicológicas, sociais e do comportamento tanto na mãe como na criança (Weissman et al., 1999). As mulheres que consomem substâncias ilícitas durante o período de gestação, podem dar origem a uma situação potencialmente geradora de efeitos disruptivos na saúde, nascimento e desenvolvimento pós-natal das crianças 
(Terplan, Garrett, \& Hartmann, 2009), bem como do estabelecimento de modalidades de interação disfuncionais da díade, nomeadamente no que se refere à inadequação de cuidados maternais e ao risco de negligência e abuso (Ferreira \& Pires, 2001).

A partir de modelos obtidos com experiências animais, é sugerido que mães que abusam de substâncias apresentam alteraçóes do seu comportamento parental, revelando-se mais agressivas a intrusivas, e que tais alteraçóes influenciam, por sua vez, o comportamento dos filhos, independentemente de terem sido expostos ou não à droga durante a gravidez (Hans, 1992, citado por Mayes \& Truman, 2002). Estão bem presentes na literatura as dificuldades das mães toxicodependentes em manter funçóes parentais organizadoras, protetoras, satisfatórias (Muchata \& Martins, 2010), bem como um ambiente de cuidados parentais adequados (Barnard \& McKeganey, 2004).

São poucos os estudos que se debruçam sobre o modo como as mães percepcionam os efeitos do abuso de substâncias sobre a sua competência parental (Mayes \& Truman, 2002). Os que existem revelam que essas mães têm consciência dos efeitos negativos do abuso de substâncias nos seus filhos, mas descrevem práticas indicadoras de que se sentem capazes enquanto mães (Baker, 1999). São muitas vezes as próprias mães que, no seio desse problema, reconhecem que perderam a capacidade de pensar "racionalmente", capacidade de tomar decisóes e fazer julgamentos, bem como a sua motivação, admitindo o impacto da sua perturbação no bem estar físico e psicológico dos filhos, quer seja através de exposição dos mesmos à violência doméstica, abuso de substâncias e outros comportamentos antissociais, quer através da perda de relaçóes significativas, receando que também os filhos venham a abusar de drogas no futuro (Haight, Carter-Black \& Sheridan, 2009).

Entendida como crise do desenvolvimento, a maternidade na mulher toxicodependente pode representar um momento-chave para o tratamento e a mudança, sendo salientada a importância e a oportunidade que a gravidez e a maternidade representam para a procura de tratamento, podendo essa alteração na vida da mulher, com o devido acompanhamento, constituir uma motivação para a alteração do padrão de consumos e estilo de vida (Davis, 2012). No entanto, não raras vezes a função materna confunde-se com o investimento narcísico (Xavier $\&$ Paúl, 1997). Diniz (1995) refere que uma situação de toxicodependência grave é, em princípio, incompatível com as exigências das funções parentais, muito mais ligada com a necessidade de ser maternalizada do que com o autêntico desejo de ser mãe. Deste modo, assiste-se muitas vezes a uma parentificação das crianças, que são investidas como prestadoras de cuidados aos seus progenitores durante o seu tratamento (Muchata \& Martins, 2010). 
Abelaira (1992) fala precisamente da dificuldade da mãe toxicodependente em sentir o seu bebé, percepcioná-lo tal como é, de ser capaz de estar atenta às reais necessidades e capacidades do bebé, embora podendo manter com o filho uma relação afetuosa intensa, que esconde no entanto prolongamentos das suas necessidades e carências afectivas. Trata-se de um sobreinvestimento de funçóes próprias do adulto e deixa esses menores em situação de fragilidade e insegurança, pois confrontam-se com a necessidade de cuidar de alguém, quando precisavam de ser cuidados (Barroso \& Salvador, 2007). Muitas vezes, essas mães têm dificuldades em encontrar um meio eficaz de manifestar a sua agressividade, deslocando esta para comportamentos de zanga e violência quer física quer verbal, para com a criança (Tucker, 1979, citado por Mayes \& Truman, 2002).

Brito (2001) realça algumas características das mães toxicodependentes e da sua relação com o bebé: o não reconhecimento da gravidez, com o consequente não seguimento desta; a falta de consciência e de sensibilidade às transformaçóes do corpo, evidenciando uma identidade feminina perturbada no contexto de uma problemática de identificação com a própria mãe; capacidade de "rêverie" materna comprometida; dificuldade de confrontação com o bebé real, visto como pouco confortante e pouco gratificante. De fato, a mãe idealiza o seu bebé como protetor e gratificante, preenchedor das suas necessidades de afecto, expetativas que rapidamente resultam frustradas ao ser confrontada com um bebé dependente e que tem necessidades, conduzindo-a, por sua vez, a sentimentos contraditórios que fazem oscilar entre comportamentos de grande proximidade ou de total abandono, verificando-se ainda a dificuldade em atribuir à criança uma dimensão própria e considerá-la como um ser individualizado. Assim, é transmitida à criança uma vivência de descontinuidade, tendo em conta que a imagem segura do objeto-mãe é transmitida pela continuidade e previsibilidade de cuidados transmitidos à criança numa interação satisfatória, sensível e afetuosa com a mãe (Brito, 2001). Têm pouca confiança nas suas capacidades como mães, mas ao mesmo tempo revelam elevadas expectativas em relação a si mesmas e ao filho, acabando por sofrer desilusóes consecutivas e sentirem-se frustradas no seu desempenho parental (Pajulo, Suchman, Kalland \& Mayes, 2006).

Os comportamentos de interação da díade mãe-criança caracterizam-se pelo empobrecimento da linguagem usada, restrição do campo de exploração da criança e pouca responsividade às suas solicitaçóes sociais (Lief, 1985, citado por Mayes \& Truman, 2002), muita atividade física e pouco envolvimento emocional, com reduzido contato do olhar. A tendência verificada nessas mães é a de ora se manterem fora da relaçáo, ora atuar fisicamente de modo intrusivo, caracterizando-se por uma aplicação da disciplina ameaçadora e autoritária (Hans, 1992, 
citado por Mayes \& Truman, 2002). A díade apresenta um padrão de regulação mútuo difícil, na medida em que a criança tem frequentemente dificuldades em regular os seus estadios de alerta, sono e stress (Molitor, Mayes \& Ward, 2003), necessitando de maior apoio parental neste domínio, e ao mesmo tempo a mãe tem maior dificuldade na leitura do comportamento do bebé, menor tolerância para comportamentos negativos da criança, bem como disponibilidade para a confortar, reatividade alterada em relação ao choro e comportamento do bebé, falhas da díade em gerir os desafios impostos pelo desenvolvimento (Sheinkopf et al., 2006), gerando-se um ciclo negativo que culmina na retirada da relação e risco aumentado de negligência e abuso (Pajulo et al., 2006). Estudos empíricos revelam que nas interaçôes mãe-criança, as mães toxicodependentes revelam menor sensitividade e envolvimento emocional com os seus filhos, são menos atentas, flexíveis e contingentes, experienciam menos prazer na interação e são mais intrusivas no seu comportamento do que as mães que não abusam de substâncias (Eiden, 2001; Johnson et al., 2002; Pajulo et al., 2006; Mayes \& Truman, 2002). As crianças filhas de pais toxicodependentes evidenciam menos emoçóes positivas durante a interação, mais respostas de stress a situaçóes novas, maiores níveis de stress como resposta a situaçōes desagradáveis, retomam de forma mais lenta a interação após interrupção, revelam menor capacidade para se manter atentas e alerta (Bendersky \& Lewis, 1998; Eiden, 2001; Eiden, Lewis, Croff \& Young, 2002; Johnson et al., 2002, Molitor et al., 2003; Goodman, Hans \& Bernstein, 2005; Flykt, Punamaki, Belt, Biringen,Salo \& Pajulo, 2012). Independentemente do género, a exposição in utero à substâncias prevê algumas perturbaçôes do comportamento que já podem ser evidentes pouco tempo após o nascimento, tornando-se estas crianças menos capazes de manter uma interação com a mãe ou menos capazes de a retomar quando interrompida (Lewis et al., 2009).

O vínculo construído entre mãe e filho é um vínculo inseguro, com tendência a ser evitante, ambivalente e desorganizado (Beeghly, Frank, Rose-Jacobs, Cabral, \& Tronick, 2003; Espinosa, Beckwith, Howard, Taylor, \& Swansan, 2001; Rodning, Beckwith, \& Howard, 1991). Estes filhos mostram, no entanto, uma capacidade adaptativa superior à que seria esperada para filhos de mães com graves dificuldades de reelaboração e organização das suas vivências pessoais (Vizzielo, Simonelli $\&$ Petenà, 2000).

As capacidades maternas de funcionamento reflexivo ("reflective functioning") correlacionam-se positivamente com um maior desenvolvimento das capacidades sociais da criança e negativamente com problemas de atenção, tendência à retirada da relação, distress materno e interações disfuncionais da díade, 
existindo alguns estudos que demonstram baixas capacidades reflexivas nas mães que abusam de substâncias (Suchman, McMahon, Slade, \& Luthar, 2005).

O abuso de substâncias ilícitas durante a gravidez é considerado um problema de saúde pública que tem potenciais efeitos de curto e longo prazo em bebés e crianças e no seu desenvolvimento (Anthony et al., 2010). A investigação documenta ocorrência de perturbações do comportamento parental em famílias toxicodependentes, medidas através da incidência de abuso físico e sexual, negligência, abandono e colocação para adopção (Black \& Mayer, 1980; Frost-Pineda, 2009; Lawson \& Wilson, 1980; Mayes \& Truman, 2002).

De facto, a negligência infantil e colocação fora do lar são muito comuns entre filhos de dependentes de opiáceos (Wilson, 1989). Uma grande percentagem de crianças, filhas de mães toxicodependentes, é colocada ou passa a viver afastada da mãe biológica. O lar dessas crianças passa a ser a casa de amigos ou familiares, ou ainda lares de adopção (Mayes \& Truman, 2002).

Existem evidências que sugerem a possibilidade das experiências de negligência e relaçóes de vinculação pobres poderem parcialmente contribuir para perturbações neurobiológicas complexas que desempenham um papel fundamental no desenvolvimento de susceptibilidade a perturbações aditivas e afetivas (Gerra et al., 2009).

Em síntese, a literatura aponta para consequências da toxicodependência na maternidade com efeitos disruptivos na saúde, nascimento e desenvolvimento pós-natal das crianças, na qualidade do vínculo e da interacção mãe-filho, bem como repercussóes que se reflectem na inadequação de cuidados maternais e risco aumentado de negligência e abuso. Frequentemente esses efeitos disruptivos estão relacionados com características psicológicas da mãe toxicodependente e com as condiçóes ambientais resultantes do contexto socioeconómico no qual se inserem.

\section{Condiçôes ambientais e famílias de origem dos pais toxicodependentes}

Verificada a incidência de abuso, negligência e a disfuncionalidade das atitudes, expetativas e comportamentos parentais nas mães que abusam de substâncias (Famularo, Kindscherff, \& Fenton, 1992; Wassermann \& Levanthal, 1993), é importante referir que existem fatores e condiçóes do contexto em que estas adultas se movem que contribuem para as dificuldades que sentem como mães (Mayes \& Truman, 2002). Um dos fatores mais importantes que pode afectar o desenvolvimento da criança e a sua adaptação é precisamente a qualidade do ambiente em que é criada (Eiden, Perterson \& Coleman, 1999). De acordo com Vizzielo et al. (2000), as comunidades em que residem as díades de mães e filhos 
atuam como mediadoras na transmissão linear da qualidade do vínculo estabelecido, e também na transmissão de um ambiente afetivo-relacional mais propício à construção de relações afectivas novas e mais adequadas, desempenhando estas comunidades e os seus técnicos um papel fundamental de contenção.

$\mathrm{Na}$ literatura são referidas: 1) as condições ambientais - condições precárias de vida e de habitação, prostituição e violência, o desemprego, o número de pessoas que toma conta da criança, 2) os antecedentes familiares que geram um padrão de continuidade multigeracional da dependência de substâncias, morte parental, abandono, discussóes e violência entre os pais, abuso de substâncias, abuso físico e sexual repetido (Mayes \& Truman, 2002), e ainda 3) as características psicológicas da mãe toxicodependente - de acordo com Hans et al. (1990, citado por Mayes \& Truman, 2002), quando à toxicodependência se adiciona a psicopatologia materna, o relacionamento nas díades torna-se ainda mais disfuncional. De facto, existem cada vez mais estudos que demonstram que as perturbações do comportamento parental em mães toxicodependentes não são resultado unicamente da toxicodependência em si, mas que variam em função de uma associação de fatores de risco multifactorial, entre os quais são de destacar a vinculação precoce das próprias mães, o índice de depressão, o grau de gravidade da toxicodependência, o meio e nível social, económico e cultural em que se inserem, bem como o suporte social percebido (Suchman et al., 2005).

De acordo com Mayes e Truman (2002) são poucos os estudos que incidem sobre os efeitos diretos da toxicodependência no comportamento parental, sendo que a maior parte se debruça sobre esse tema considerando o abuso de substâncias uma variável mediadora. $\mathrm{Na}$ verdade, está pouco esclarecido quais as próprias consequências da toxicodependência a nível individual, diferenciando-as de outras variáveis, tais como o estatuto e ambiente sócio económico. Por exemplo, de acordo com Latvala et al. (2009), uma consequência direta do abuso de substâncias nas capacidades cognitivas é a lentificação psicomotora, que surge independentemente de outros fatores de risco. Outras capacidades, tais como as intelectuais verbais surgem já como mediadas pelo baixo estatuto socioeconómico e grau de instrução.

Habitualmente, a exposição a substâncias é relacionada com consequências negativas na criança, mas é importante ter em conta que algumas dessas consequências negativas podem advir de relaçóes de vinculação inseguras e baixo nível de qualidade na prestação de cuidados, mais do que a exposição à substâncias em si, colocando também em relevo o fator exposição a ambiente sociocultural de risco (Bergin \& McCollough, 2009).

Vários fatores constituem-se como fatores de stress ambiental, com impacto no desenvolvimento da criança que nasce numa família em que um ou mais 
membros é toxicodependente (Holley, 2009). Podem ser considerados alguns fatores intervenientes no desenvolvimento da criança, tais como as influências genéticas, o estado nutricional da mãe, pobreza e fatores de stress associados, perturbação mental, quer como factor predisponente à toxicodependência, quer como consequência do uso de drogas, doenças infetocontagiosas e deficiente qualidade/ausência de prestação de cuidados.

A transmissão familiar intergeracional de comportamentos aditivos é testemunhada por diversos estudos (Brook et al., 2002; Cadoret, Yates, Troughton, Woodworth, \& Stewart, 1995; Conray, Flay, \& Hill, 1992; Merykangas et al., 1998 , citados por Walden, Iacono \& McGue, 2007), nomeadamente a transmissão da vulnerabilidade na exteriorização de comportamentos (Verona \& Sachs-Ericsson, 2005). Embora se verifique que determinadas características e vulnerabilidades da personalidade são herdadas geneticamente (Kendler, Karkowski, Neale, \& Prescott, 2000; Kendler \& Prescott, 1999; True et al., 1999), o meio familiar é muito importante como fator preditivo de envolvimento em comportamentos de abuso de substâncias na adolescência (Catalano, Haggerty, Gainey \& Hoppe, 1997; Stanger et al., 2002; Walden, Iacono, \& McGue, 2007). Também a psicopatologia de um ou de ambos os pais, em famílias em que um dos pais é toxicodependente, pode constituir um fator de risco agravado para o surgimento de perturbaçóes na adolescência (Stanger et al., 2002).

Os fatores de risco parentais ou familiares incluem práticas de gestão familiar pobres, conflitos familiares, atitudes parentais favoráveis e permissivas no que se refere ao consumo de drogas e envolvimento dos filhos nas próprias práticas parentais de abuso de substâncias (Catalano et al., 1997).

$\mathrm{O}$ abuso de drogas no período pré-natal encontra-se associado à percepção maternal de baixa qualidade de vida, maior predisposição de abuso de substâncias por parte de familiares e amigos e a riscos de ordem psicossocial, com eventuais consequências tais como comportamento parental e prestação de cuidados desadequados, repercutindo em dificuldades psicológicas e do desenvolvimento nos filhos (Derauf et al., 2007). As crianças filhas de pais toxicodependentes crescem frequentemente em contextos sociais caracterizados por problemas relacionais, desemprego, questões relacionadas com abuso e implicação de serviços de proteção de menores e uma incidência relativamente alta de perturbação mental parental, sendo que uma grande percentagem dessas crianças apresenta problemas psicossociais semelhantes na sua vida adulta. De facto, mais de cinquenta por cento dos pais que abusam de substâncias repetem os mesmos padróes de vinculação, comportamentos e atitudes com os quais conviveram na infância (Söderstrom \& Skarderud, 2009). 
Alguns fatores de proteção podem ser uma vinculação sólida e afetuosa, bom suporte parental das competências dos filhos, interação e comunicação positiva entre pais e filhos (Catalano et al., 1997), pelo menos um modelo positivo e saudável de relação na infância, mudanças na autopercepção através da criação de relaçóes durante o tratamento, capacidade de gerir sentimentos ambivalentes, modelos positivos de parentalidade, contacto com exemplos de pessoas com resultados positivos na sua recuperação, desejo de ser mãe e de autoconhecimento e desenvolvimento pessoal (Metz, 2009). Mães que se atingem os seus objetivos de tratamento têm mais tempo de abstinência do uso de drogas, emprego estável e salários mais elevados, apoio do companheiro e têm também mais probabilidade de se manterem a viver com os filhos (Grant et al., 2011).

Em síntese, pode dizer-se que os fatores ambientais e contextuais nos quais as mães toxicodependentes se inserem constituem-se como variáveis mediadoras importantes que concorrem para um comportamento parental desadequado com repercussões ao nível da qualidade dos cuidados prestados, relação da díade e problemas de saúde e desenvolvimento nas crianças. A importância destes fatores reflete-se também na sua transmissão intergeracional em que padrões de vinculação, comportamentos e atitudes são transmitidos de geração em geração.

\section{Conclusão}

De acordo com a literatura corrente a toxicodependência tendencialmente implica consequências e interferências na capacidade para ser mãe ou ser pai. São referidos efeitos disruptivos na saúde, nascimento e desenvolvimento pós-natal das crianças, na qualidade do vínculo e da interação mãe-filho, bem como repercussóes que se refletem na inadequação de cuidados maternais e risco aumentado de negligência e abuso. Frequentemente esses efeitos disruptivos estão relacionados com características psicológicas da mãe toxicodependente e com as condições ambientais resultantes do contexto socioeconómico no qual se inserem.

Consistindo a parentalidade na capacidade de providenciar os cuidados necessários ao desenvolvimento físico e psicológico da criança, considerando-a na sua individualidade e no momento particular do desenvolvimento em que se encontra, quando o comportamento parental não responde de forma adequada às solicitaçóes da criança pode representar um risco para a sua saúde física e psicológica. Uma dessas situaçóes potencialmente disruptivas é exatamente a da toxicodependência na maternidade, em que a maior parte dos estudos consideram o comportamento parental em toxicodependentes, perturbado, deficiente, frágil e com 
consequências nefastas para a criança e sua relação com os pais. Os pais revelam-se menos centrados nas crianças e mais em si próprios, têm dificuldades no controlo das emoções, são menos sensíveis, responsáveis e afetuosos, são mais negligentes com as necessidades físicas dos seus filhos e muitas vezes criam-se situações em que a criança fica separada de um dos pais ou de ambos (Muchata \& Martins, 2010)

Constatada a enorme importância do papel dos pais e das relaçóes precoces no desenvolvimento físico e psicológico equilibrado dos filhos, e dada a escassez de trabalhos que retratem a experiência e as dificuldades sentidas pelas mães toxicodependentes no contexto do comportamento parental e que possam servir de base a uma intervenção mais adaptada, torna-se importante aprofundar como é sentida e gerida uma situação que é considerada uma situação de risco, potencialmente disruptiva, não só para a criança, como para a própria mãe.

A investigação tem focalizado a atenção em algumas áreas do comportamento parental, tais como as interaçóes no contexto da alimentação, desenvolvimento social, atividades didáticas e estabelecimento de limites (Mayes \& Bornstein 1997). Os poucos estudos realizados sobre as interaçóes sociais entre mães toxicodependentes e os seus filhos não identificam se a mãe é a principal cuidadora, qual o papel do suporte social na sua vida e no auxílio a lidar com as dificuldades, como ocorre este suporte e em que momentos dos seus percursos, bem como interfere com os outros fatores intervenientes, quais as condiçóes em que vivem as famílias dessas mães, quantos adultos cuidam da criança, qual a estrutura familiar, entre outros (Mayes \& Bornstein, 1997). É de extrema importância que a investigação abranja esses e outros aspetos que permanecem pouco claros e que muito poderão contribuir para perceber como o abuso de substâncias pode influenciar o comportamento parental. $\mathrm{Na}$ verdade, os efeitos biológicos diretos do abuso de substâncias, assim como os fatores ambientais e contextuais que podem agravar as consequências nas crianças e relação mães-filhos, são contemplados de forma lacunar e incongruente na literatura, resultando em dificuldades na distinção entre os múltiplos fatores intervenientes (Kim \& Krall, 2006). Faltam estudos que contemplem como a toxicodependência afeta as atividades quotidianas de mães e crianças, tidas como mediadoras do desenvolvimento social, afetivo e cognitivo precoce (uso da linguagem materna, encorajamento de comportamentos de exploração, direcionamento da atenção, atenção conjunta, referências sociais); que expliquem quais são os fatores que fazem variar a probabilidade de risco de disfunções na díade e de disrupção da relação, abuso e negligência; que refiram a contribuição de fatores de morbilidade que permitam prever a toxicodependência e a adequação do comportamento parental; debruçar-se sobre os fatores de proteção que permitem à mãe toxicodependente desenvol- 
ver uma relação harmoniosa e competências parentais adequadas, apesar do seu problema de abuso de substâncias.

Apesar de a investigação de Mayes \& Truman (2002) ao longo das duas últimas décadas apontar já essas lacunas, verificamos que a investigação tem-se focado sobretudo em medidas indiretas do comportamento parental através de estudos de perturbaçóes do comportamento e do desenvolvimento sócio-emocional de crianças filhas de mães toxicodependentes, faltando dados sobre a verdadeira relação direta entre abuso de substâncias e disfunções familiares e da parentalidade, bem como a experiência e dificuldades inerentes à maternidade nesta situação de risco, sendo ainda de referir a escassez e necessidade de estudos longitudinais que permitam avaliar essas situações ao longo do tempo, tão importantes para a construção de programas de intervenção adequados e eficazes (Fiks, Johnson, \& Rosen, 1985).

Em termos de intervenção, as revisões de literatura atuais apontam para a existência de uma quantidade enorme de metodologias de intervenção com enfoques variados e momentos diversos de ação, embora se encontre reduzida publicação científica acerca da testagem da sua eficácia, que contemple uma avaliação sistemática de resultados. Verifica-se, assim, uma falta de sistematização considerável, sendo muitíssimo importante o desenvolvimento de estudos que permitam uma comparação dos modelos de intervenção já existentes na sua eficácia aos diferentes níveis em que pretendem intervir; de igual modo, os estudos deverão também debruçar-se sobre fatores pouco abordados como são os obstáculos que as grávidas e mães toxicodependentes percepcionam e se constituem como verdadeiras barreiras ao sucesso da intervenção, tais como a percepção de não precisar de tratamento, medo de ações punitivas por parte dos técnicos, obstáculos percebidos no próprio programa de tratamento, os parceiros, mudanças no estatuto de toxicodependente e de grávida (Jessup, Humphreys, Brindis \& Lee, 2003), a forma como a pressão externa pode influenciar a trajectória da toxicodependência da grávida ou mãe (Davis, 2012). Contudo, até a investigação se debruçar sobre aspectos mais específicos da interação mãe-criança no contexto da toxicodependência, continuarão a existir aspectos não clarificados. Esses estudos são fundamentais para promover uma intervenção efetiva e específica, adaptada clinicamente a cada caso e a cada contexto (Kim \& Krall, 2006; Mayes \& Truman, 2002). A intervenção deve, sobretudo, agir para além dos mitos, recusar a imagem e os argumentos que referem as mães toxicodependentes como "más mães" para, assim poder combater a criação e manutenção de práticas que estigmatizam, mais do que fazem algo de útil por essas mães e seus filhos (Hayford, Epps \& Dahl-Regis, 1988). 


\section{Referências}

Abelaira, R. (1992). Relação mãe-filho toxicodependente. Colectânea de Textos do Centro das Taipas, 5, 73-75.

Anthony, E., Austin, M., \& Cormier, D. (2010). Early detection of prenatal substance exposure and the role of child welfare. Children and Youth Services Review, 32(1), 6-12.

Baker, P. (1999). "I take care of my kids" - Mothering practices of substance-abusing women. Gender \& Society, 13(3), 347-363.

Barnard, M., \& McKeganey, N. (2004). The impact of parental problem drug use on children: What is the problem and what can be done to help? Addiction, 99(5), 552-559.

Barroso, C., \& Salvador, E. S. (2007). Crianças que parecem andar um pouco por aí, pelo ar, ... Os filhos dos toxicodependentes no CAT de Leiria e no Pólo da Marinha Grande. Toxicodependências, 13(3), 61-68.

Beeghly, M., Frank, D. A., Rose-Jacobs, R., Cabral, H., \& Tronick, E. (2003). Level of prenatal cocaine exposure and infant-caregiver attachment behavior. Neurotoxicology and Teratology, 25(1), 23-38.

Bendersky, M., \& Lewis, M. (1998). Arousal modulation in cocaine-exposed infants. Developmental Psychology, 34(3), 555-564.

Bergin, C., \& MsCollough, P. (2009). Attachment in substance-exposed toddlers: The role of caregiving and exposure. Infant Mental Health Journal, 30(4), 407-423.

Black, R., \& Mayer, J. (1980). Parents with special problems: Alcoholism and opiate addiction. Child Abuse and Neglect, 4, 45-54.

Brito, I. (2001). Continuidades na maternidade da toxicodependente. Toxicodependências, $7(3), 79-82$.

Catalano, R. F., Haggerty, K. P., Gainey, R. R., \& Hoppe, M. J. (1997). Reducing parental risk factors for children's substance misuse: preliminary outcomes with opiate-addicted parents. Substance Use and Misuse, 32(6), 699-721.

Davis, K. J. (2012). Making lemonade out of lemons: A case report and literature review of external pressure as an intervention with pregnant and parenting substance-using women. Journal of Clinical Psychiatry, 73(1), 51-56.

Derauf, C., LaGasse, L., Smith, L., Grant, P., Shah, R., Arria, A., ... Lester, B. (2007). Demographic and psychosocial characteristics of mothers using methamphetamine during pregnancy: Preliminary results of the infant development, environment and lifestyle study (IDEAL). The American Journal of Drug and Alcohol Abuse, 33(2), 281-289.

Diniz, J. S. (1995). A mãe toxicodependente e o seu bebé. Toxicodependências, 1, 67-76.

Eiden, R. (2001). Maternal substance use and mother-infant feeding interactions. Infant Mental Health Journal, 22(4), 497-511. 
Eiden, R. D., Lewis, A., Croff, S., \& Young, E. (2002). Maternal cocaine use and infant behavior. Infancy, 3(1), 77-96.

Eiden, R. D., Peterson, M., \& Coleman, T. (1999). Maternal cocaine use and the caregiving environment during childhood. Psychology of Addiction Behaviors, 13(4), 239-302.

Espinosa, M., Beckwith, L., Howard, J., Tyler, R., \& Swanson, K. (2001). Maternal psychopathology and attachment in toddlers of heavy cocaine-using mothers. Infant Mental Health Journal, 22, 316-333.

Famularo, R., Kinscherff, R., \& Fenton, T. (1992). Parental substance abuse and the nature of child maltreatment. Child Abuse and Neglect, 16, 475-483.

Ferreira, A. P., \& Pires, A. (2001). Toxicodependência materna e comportamento parental. In A. Pires (Ed.), Crianças (e pais) em risco (pp. 303-321). Lisboa: Instituto Superior de Psicologia Aplicada.

Fiks, K. B., Johnson, H. L., \& Rosen, T. S. (1985). Methadone-maintained mothers: 3-year follow-up of parental functioning. The International Journal of the Addictions, 20(5), 651-660.

Flykt, M., Punamaki, R., Belt, R., Biringen, Z., Salo, S., \& Pajulo, M. (2012). Maternal representations and emotional availability among drug-abusing and nonusing mothers and their infants. Infant Mental Health Journal, 33(2), 123-138.

Frost-Pineda, K. (2009). Addiction and pregnancy intentions: Understanding the why behind the what. Dissertation Abstracts International Section A: Humanities and Social Sciences, 69(7-A), 2766.

Gerra., G., Leonardi, C., Cortese, E., Zaimovic, A., Dell'Agnello, G., Manfredini, M., ... Donnini, C. (2009). Childhood neglect and parental care perception in cocaine addicts: Relation with psychiatric symptoms and biological correlates. Neuroscience and Biobehavioral Reviews, 33(4), 601-610.

Goodman, G., Hans, S., \& Bernstein, V. (2005). Mother expectation of bother and infant attachment behaviors as predictors of mother and child communication at 24 months in children of methadone - maintained women. Infant Mental Health Journal, 26(6), 549-569.

Guerreiro, C. (2001). Reflexóes sobre a mulher toxicodependente e a gravidez: a mulher toxicodependente e o planeamento familiar, a gravidez e a maternidade. Lisboa: Edição da Fundação Nossa Senhora do Bom Sucesso.

Grant, T., Huggins, J., Graham, C., Ernst, C., Whitney, N., \& Wilson, D. (2011). Maternal substance abuse and disrupted parenting: Distinguishing mothers who keep their children from those who do not. Children \& Youth Services Review, 33(11), 2176-2185.

Haight, W., Carter-Black, J., \& Sheridan, K. (2009). Mothers' experience of methamphetamine addiction: A case-based analysis of rural, Midwestern women. Children and Youth Services Review, 31(1), 71-77. 
Hayford, S. M., Epps, R. P., \& Dahl-Regis, M. (1988). Behavior and development patterns in children born to heroin-addicted and methadone-addicted mothers. Journal of the National Medical Association, 80(11), 1197-2000.

Holley, M. (2009). Fetal, neonatal, and early childhood effects of prenatal methamphetamine exposure. The Praeger international collection on addictions, 2, 351-370.

Jessup, M. A., Humphreys, J. C., Brindis, C. D., \& Lee, K. A. (2003). Extrinsic barriers to substance abuse treatment among pregnant drug dependent women. Journal of Drug Issues, 33(2), 285-304.

Johnson, A. L., Morrow, C. E., Accornero, V. H., Xue, L., Anthony, J. C., \& Bandstra, E. S. (2002). Maternal cocaine use: Estimated effects on mother-child play interactions in the preschool period. Journal of Developmental \& Behavioral Pediatrics, 23, 191-203.

Kendler, K. S., \& Prescott, C. A. (1999). A population-based twin study of lifetime major depression in men and women. Archives of General Psychiatry, 56(1), 39-44.

Kendler, K. S., Karkowski, L., Neale, M., \& Prescott, C. (2000). Illicit psychoactive substance use, heavy use, abuse, and dependence in a US population-based sample of male twins. Archives of General Psychiatry, 57, 261-269.

Kim, J., \& Krall, J. (2006). Literature Review: Effects of Prenatal Substance Exposure on Infant and Early Childhood Outcomes. Berkeley, CA: National Abandoned Infants Assistance Resource Center - University of California at Berkeley.

Latvala, A., Castaneda, A. E., Perälä, J., Saarni, S. I., Aalto-Setälä, T., Lönnqvist, J., ... Tuulio Henriksson, A. (2009). Cognitive functioning in substance abuse and dependence: a population-based study of young adults. Addiction, 104(9), 1558-1568.

Lawson, M., \& Wilson, G. (1980). Parenting among women addicted to narcotics. Child Welfare, 59, 67-79.

Lewis, M., Phillips, G., Bowser, M., DeLuca, S., Rosen, T., \& Johnson, H. (2009). Cocaine-exposed infant behavior during still-face: risk factor analyses. American Journal of Orthopsychiatry, 79(1), 60-70.

Limede, R. (2014). A transição inesperada para a maternidade: um estudo com mães estudantes e mães toxicodependentes. (Tese de Mestrado. Faculdade de Psicologia e Ciências da Educação da Universidade de Coimbra, Coimbra).

Mayes, L. (2002). A behavioural teratogenic model of the impact of prenatal cocaine exposure on arousal regulatory systems. Neurotoxicology-and-Teratology, 24(3), 385-395.

Mayes, L., \& Bornstein, M. (1997). The development of children exposed to cocaine. In S. Luthar, J. Burack, D. Cicchetti, \& J. Weisz (Eds.), Development psychopathology: perspectives on adjustment, risk and disorder (pp. 166-168). Cambridge: Cambridge University Press.

Mayes, L. C., \& Truman, S. D. (2002). Substance abuse and parenting. In M. H. Bornstein (Ed.), Handbook of parenting - Volume 4: Social conditions and applied psychology (pp. 329360). Mahwah, NJ: Erlbaum. 
Metz, H. (2009). Life stories of substance abusing mothers: Assessing potential protective factors in becoming drug free. The Sciences and Engineering, 70(5-B), 3179.

Molitor, A., Mayes, L. C., \& Ward, A. (2003). Emotion regulation behavior during a separation procedure in 18-month-old children of mothers using cocaine and other drugs. Development and Psychopathology, 15, 39-54.

Muchata, T., \& Martins, C. (2010). Impacto da toxicodependência na parentalidade e saúde mental dos filhos - Uma revisão bibliográfica. Toxicodependências, 16(1), 47-56.

Pajulo, M., Suchman, N., Kalland, M., \& Mayes, L. (2006). Enhancing the effectiveness of residential treatment for substance abusing pregnant and parenting women: focus on maternal reflective functioning and mother-child relationship. Infant Mental Health Journal, $27(5), 448-465$.

Pires, A. (2001). Crianças e pais em risco. Lisboa: ISPA.

Rodning, C., Beckwith, L., \& Howard, J. (1991). Quality of attachment and home environments in children prenatally exposed to PCP and cocaine. Development and Psychopathology, 3, 351-366.

Sheinkopf, S., Lester, B., LaGasse, L., Seifer, R., Bauer, C., Shankaran, S., ... Wright, L. (2006). Interactions between maternal characteristics and neonatal behavior in the prediction of parenting stress and perception of infant temperament. Journal of Pediatric Psycho$\log y, 31(1), 27-40$.

Söderström, K., \& Skarderud, F. (2009). Minding the baby - Mentalization-based treatment in families with parental substance use disorder: theoretical framework. Nordic Psychology, 61(3), 47-65.

Stanger, C., Kamon, J., Dumenci, L., Higgins, S. T., Bickel, W. K., Grabowski, J., \& Amass, L. (2002). Predictors of internalizing and externalizing problems among children of cocaine and opiate dependent parents. Drug and Alcohol Dependence, 66(2), 199-212.

Suchman, N., McMahon, T., Slade, A., \& Luthar, S. (2005). How early bonding, depression, and perceived support work together to influence drug-dependent mothers' caregiving. American Journal of Orthopsychiatry, 75(3), 431-445.

Terplan, M., Garrett, J., \& Hartmann, K. (2009). Gestational age at enrollment and continued substance use among pregnant women in drug treatment. Journal of Addictive Diseases, 28 (2), 103-112.

True, W, R., Scherrer, J. F., Xian, H., Lin, N., Eisen, S. A., Lyon, M. J., ... Tsueng, M. T. (1999). Interrelationship of genetic and environmental influences on conduct disorder and alcohol and marijuana dependence symptoms. American journal of Medical Genetics, 88, 391-397.

Verona, E., \& Sachs-Ericsson, N. (2005). The intergenerational transmission behaviors in adult participants: the mediating role of childhood abuse. Journal of Consulting and Clinical Psychology, 73(6), 1135-1145. 
Vizzielo, G. F., Simonelli, A., \& Petenà, I. (2000). Representaciones maternas y transmisión de los factores de riesgo y protección en hijos de madres drogodependientes. Adicciones, 12(3), 413-424.

Xavier, M. R., \& Paúl, M. C. (1997). Avaliação do estatuto de risco de um grupo de crianças de dois anos expostas a drogas ilícitas no período pré-natal. In J. L. P. Ribeiro (Ed.), Actas do $2^{\circ}$ Congresso Nacional de Psicologia da Saúde (pp. 449-464). Lisboa: ISPA

Walden, B., Iacono, W. G., \& McGue, M. (2007). Trajectories of change in adolescent substance use and symptomatology: Impact of paternal and maternal substance use disorders. Psychology of Addictive Behaviors, 21(1), 35-43.

Wassermann, D. R., \& Leventhal, J. M. (1993). Maltreatment of children born to cocaine-dependent mothers. American Journal of Diseases of Children, 147, 1324-1328.

Weissman, M. M., McAvay, G., Goldstein, R. B., Nunes, E. V., Verdeli, H., \& Wickramaratne, P. J. (1999). Risk/protective factors among addicted mothers' offspring: a replication study. American Journal of Drug and Alcohol Abuse, 25(4), 661-679.

Wilson, M. N. (1989). Child development in the context of the Black extended family. American Psychologist, 44(2), 380-385.

\section{Agradecimentos}

Esta investigação foi apoiada pela Fundação para a Ciência e a Tecnologia, Bolsa de Doutoramento SFRH/ BD/ 36907/ 2007, atribuída a Sofia A. Silva.

Recebido em 23 de janeiro 2014 Aceito para publicação em 15 de agosto de 2014

DOI: 10.1590/0103-56652015000100005 